\title{
The KIT Swiss Knife Gripper for Disassembly Tasks: A Multi-Functional Gripper for Bimanual Manipulation with a Single Arm
}

\author{
Júlia Borràs, Raphael Heudorfer, Samuel Rader, Peter Kaiser and Tamim Asfour
}

\begin{abstract}
This work presents the concept of a robotic gripper designed for the disassembly of electromechanical devices that comprises several innovative ideas. Novel concepts include the ability to interchange built-in tools without the need to grasp them, the ability to reposition grasped objects in-hand, the capability of performing classic dual arm manipulation within the gripper and the utilization of classic industrial robotic arms kinematics within a robotic gripper. We analyze state of the art grippers and robotic hands designed for dexterous in-hand manipulation and extract common characteristics and weak points. The presented concept is obtained from the task requirements for disassembly of electromechanical devices and it is then evaluated for general purpose grasping, in-hand manipulation and operations with tools. We further present the CAD design for a first prototype.
\end{abstract}

\section{INTRODUCTION}

Currently, electronic products have a short life cycle, leading to large amounts of very diverse end-of-use devices. Studies like [1] show that recycling processes of these products are either highly manual or, when fully automated, destructively separate parts and components. The resulting heterogeneous mixtures make the isolation of individual materials, potentially precious or dangerous, hard. Current processes allow the recovery of the most expensive materials like gold, silver and copper. However, fully automated processes could potentially allow to recover aluminum, iron, cobalt and nickel which are usually lost in the current recycling processes [2], [3]. Automated disassembly processes would further ease the proper management of toxic materials such as lead, mercury, cadmium, and chromium that are present in electronic products. In this context, there is an increasing need for research on automated disassembly of electromechanical devices [1], [3].

This paper is part of the research for the European project IMAGINE, that studies how robots can learn disassembly tasks, e.g. from human demonstration. Automated recycling processes need to be flexible enough to deal with the diversity of the technological industry. Therefore, the ability of robots to autonomously learn how to properly disassemble different devices is crucial. However, disassembly tasks are

The research leading to these results has received funding from the European Union H2020 Programme under grant agreement no. 731761 (IMAGINE) and from the European Research Council (ERC) from the European Union Horizon 2020 Programme under grant agreement no. 741930 (CLOTHILDE).

The authors are with the Institute for Anthropomatics and Robotics, Karlsruhe Institute of Technology, Karlsruhe, Germany, asfourekit.edu Currently, Júlia Borràs is with Institut de Robòtica i Informàtica Industrial, Barcelona, Spain jborraseiri.upc.edu

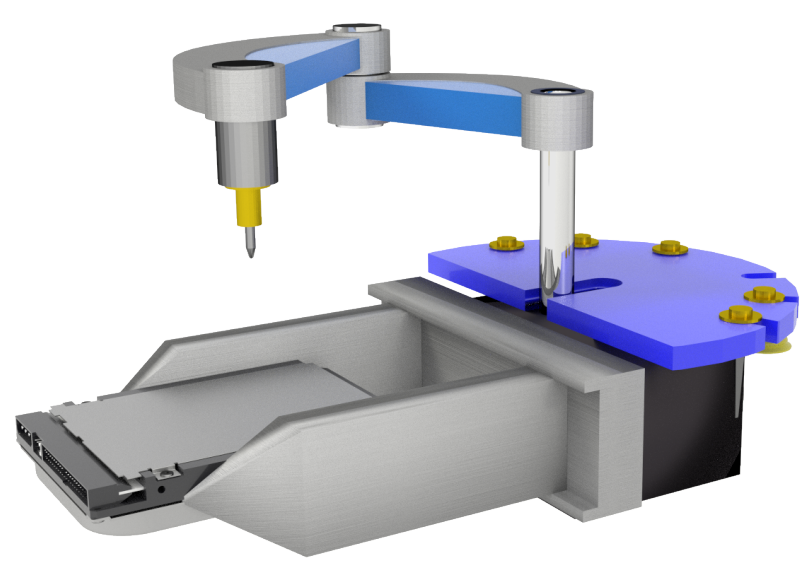

Fig. 1: The KIT multi-functional gripper performing the disassembly of a hard disk. The gripper concept allows the exchange of built-in end-effector tools to operate on grasped objects.

challenging for robots because they involve highly precise bimanual manipulations and require handling different tools in rapid succession. Furthermore, with state of the art grippers, rapid tool changes can severely disrupt the flow of operation. Most industrial solutions are ad-hoc and avoid the use of multi-functional grippers [2]. To our best knowledge, most of the grippers designed for disassembly are highly specialized for specific products and none of them solves the tool change problem.

We present a novel gripper concept for disassembly tasks devised for performing bimanual manipulation with a single arm and autonomous tool change. The gripper is designed for disassembly tasks, but we are also interested in general grasping capabilities and in performing in-hand dexterous manipulation. One of the main ideas of the proposed concept is that it has built-in tools, inspired by Swiss knives, and combines characteristics from dual arm manipulation and gripper designs. Another novelty is that the gripper is designed to avoid the necessity of bimanual manipulations in situations where one hand just holds an object in place and the second hand is used to perform the task. Our gripper can perform such bimanual manipulations using a single arm, which can simplify the robot structure, increase the precision in object space [4] and improve a dual arm system to utilize the second arm for something more useful than 
just holding an object in place. In this work, we will show how we designed the gripper, shown in Fig. 1, for grasping different objects, for performing several manipulations on the object, including in-hand re-grasp reorientation; and in-hand complex manipulations using the built-in tools.

This paper is organized as follows. Section II reviews works on gripper designs for dexterous in-hand manipulation and disassembly tasks. Section III details the task requirements and introduces the detailed concept of the proposed gripper. Section IV shows how we plan to satisfy the task requirements with the proposed concept. Section V presents detailed specifications and a CAD design for the implementation of a prototype. Finally, Section VI and VII discuss and summarize the conclusions and future work.

\section{RELATED WORK}

\section{A. Bimanual manipulation vs. in-hand manipulation}

Classic research on robotic hands and grippers studies the trade-offs between simplicity, generality and performance. Simplicity means few actuators, few and simple sensors and the absence of complicated mechanics [5], generality refers to the ability to grasp different objects, and performance indicates accuracy, speed, repeatability, gripping strength and robustness [6]. We want to point out that none of these parameters refer to the ability for dexterous in-hand manipulation, a quality that is of growing importance. Both complex human-like hands and simpler underactuated ones fail in performing dexterous tasks such as sophisticated disassembly tasks, e.g. unscrewing or levering. We believe we should extend the concept of generality to include not only grasping, but also the ability to perform as many manipulation tasks as possible in-hand. In this case, we need to consider an extra trade-off between in-hand manipulation ability and grasping generality [7].

The dexterous manipulation capabilities of existing robotic hands and grippers in the literature are limited. However, if dual-arm robotic systems are considered as single manipulators, examples for dexterous manipulation exist. Works like [8] and [4] pointed out dualities between in-hand manipulation and bimanual manipulation, listing grippers as examples of dual arm systems. A prominent example is [9] where several gripper designs are introduced with different tools at the fingertips. In this direction, we believe that non-humanoid grippers could greatly benefit from more dexterous kinematic structures.

We further believe that the capabilities of bimanual robotic systems could be enhanced by the development of grippers that can perform classical dual arm manipulation actions inhand. A prominent pattern of dual arm manipulation consists in one arm that holds an object in place, while the other arm performs the manipulation action [8], [10]. In these cases, the hand that holds the object, no mater how sophisticated it is, has a similar function as a parallel-jaw gripper. In addition, manipulating an object using a whole arm is not always advantageous: In comparison to larger arms, capable dexterous hands are potentially faster and more precise for small movements in the object space [4]. Therefore, if one of the arms of the dual arm system has a gripper capable of performing both the task of holding the object and manipulating it the dexterity of the second hand could be utilized in a more advantageous way.

\section{B. Grippers for in-hand manipulation}

There are a few examples of gripper designs that explore alternative kinematics to enhance in-hand manipulation. For instance, [11] proposed a gripper with four cylindrical fingers with 4 DoF each, consisting of a 2 DoF 5-linkage mechanism to open and close, a prismatic joint to extend their length and the ability to rotate around their self axis. This allows for relevant in-hand manipulation like rotating the object after being grasped at the expense of needing 16 actuated DoF. Our design has $6 \mathrm{DoF}$ and it is capable of complex in-hand manipulation.

Other works like [12] propose an interesting design where two flat independent fingers can close as a parallel jaw. The fingers are equipped with two turning disks at the tips allowing manipulation by rolling objects inside the hand or on a table. In addition to the dexterous manipulations, the gripper can also work as a parallel gripper. A similar idea was explored in [9].

In a more functional direction, [13] presents a design that combines two simple underactuated grippers that can work in collaboration to achieve in-hand object reorientation by performing finger gaits. In this direction, it is interesting to point out that an increasing amount of designs include the idea of gripper of grippers to be able to perform dexterous in-hand manipulation as well as flexible grasps [13], [14], [9], [15].

Finally, we observe that gripper fingers usually have symmetric geometries, meaning similarly shaped and symmetrically distributed around the palm. The rationale is that grasping involves symmetric movement where all fingers close around the object. However, such symmetry may not always be optimal for in-hand manipulation [7]. We introduce the idea that in one robotic hand some fingers can be devoted to grasping while others are responsible for performing manipulation actions, having completely different kinematic structures. The proposed design is inspired in typical industrial assembly systems in an attempt to bring the high performance and manipulation capabilities inside the hand.

\section{Grippers for assembly and disassembly tasks}

Assembly and disassembly tasks require a sophisticated level of precise, complex manipulation [16]. It is therefore interesting to review grippers that are specifically designed for assembly and disassembly, even if they are specialized to particular products.

The gripper in [17] is a parallel gripper with 2 phalanges per finger that adapt to rectangular and cylindrical pieces of a washing machine, plus another finger to secure pieces that need to be assembled. The gripper phalanges are particularly designed with slots that fit exactly with protrusions of the pieces that have to be grabbed. More flexibility is present 


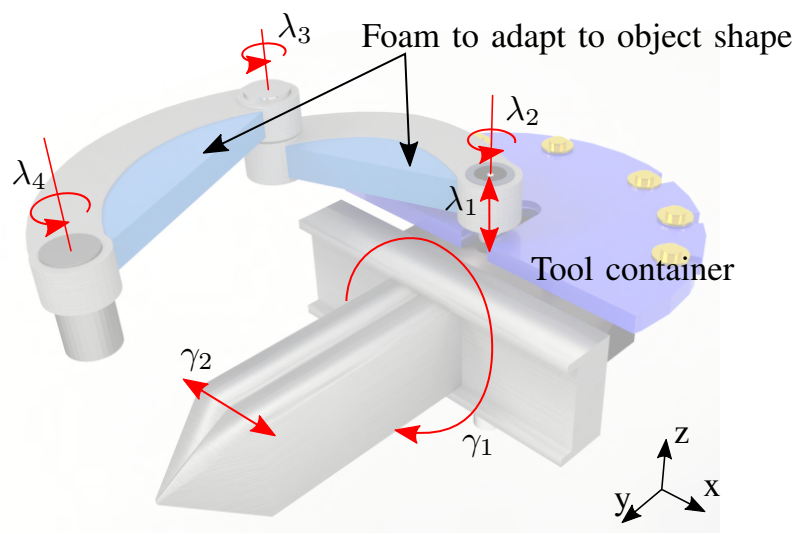

Fig. 2: Kinematic structure of the KIT Swiss Knife Gripper.

in the design of [14] for performing precise grasping of differently shaped batteries using two parallel jaw grippers, one of them fixed and the other build on a spindle axis that can translate and rotate. This is an example of the gripper of grippers concept mentioned above. Both jaw grippers are specifically designed to grab only the arresters of batteries, but the design could be capable of many types of in-hand manipulation in another scenarios. Seliger et al. [18] propose a gripper where one of the fingers is a smart screwdriver that can unscrew independently of the screw head geometry. The gripper has two additional needle fingers that are used to fix the object that needs to be unscrewed. The main drawback is that the needles can destroy the object and therefore, it may not be able to perform a complete disassembly process. Another way of achieving generality is using reconfigurable structures and modularity. This is the case of the gripper in [15] where every finger is autonomous, has $2 \mathrm{DoF}$, two phalanges and it is equipped with electromagnetic grippers at the fingertip. The gripper can be used with 1,2, 3 or more fingers.

Other solutions for disassembly task involve full robot arms [19], [20]. Particularly interesting is the work [20], [21], that presents a robot arm equipped with a screwdriver tool capable of autonomously changing bits. To the best of the authors' knowledge, no other existing gripper design addresses the particular issue of tool handling or an optimized method to grab different tools, besides the ability of grasping tools as any other object. However, many works point out the necessity of providing a tool change system [18], [14], [16] for disassembly tasks.

\section{CONCEPT OF THE MUTI-FUNTIONAL GRIPPER}

\section{A. Extracting requirements from tasks}

A list of basic actions has been compiled from the observation of videos of human operators performing disassembly of hard drives. We have classified all the actions into three categories:

1) Grasping actions

- Before grasping: Picking up, grasping

- After grasping: Dropping, placing
- During grasping: Rattling, shaking, banging (hit with hammer), breaking off (first grasp the piece to break off, then pull away)

\section{2) Pushing actions:}

- Pushing, pressing something down

\section{3) Actions with tools}

- Pulling (with a hook tool), unscrewing, levering, scraping.

Note that the list of actions may not be complete for other disassembly tasks. We observed that actions of grasping are commonly implemented by many grippers, but the use of tools can constitute a challenging task if we consider that a disassembly process includes different action with different tools in rapid succession. Therefore, one of the design requirements is to have a smart tool change system that allows a fast tool change process. Considering that the weakest points in a gripper are usually the finger joints, another desired design objective is the consideration of joint-less fingers for pushing actions that require strength and robustness. In addition, when performing actions in an object, e.g. a piece to be disassembled, usually bimanual manipulation is required mainly to hold the object in place, requiring a robotic system with two arms, that tend to be much more expensive and complex than a single arm configuration. As an alternative, the object can be fixed on the table. However, this limits the flexibility of the system, as the process may require to change the orientation of the object during the process. Therefore, we are aiming at a versatile gripper, which is also capable of fixating the object that is working on by itself.

To realize the grasping actions, we analyzed grippers with two and three fingers for high precision and robustness. Finally, electromechanical devices can be of very diverse shapes and sizes, but we restrict ourselves for a preliminary design to middle sized electronics such as hard-drives, $C D$ readers, smartphones or similarly sized electronic devices. In addition, the gripper should be able to grasp small sized objects such as screws of different sizes and odd-shaped disassembled pieces. However, such grasps can be achieved with one of the tools designed for small grasps.

\section{B. Concept proposal}

As a result of the discussion in the previous section, we propose a gripper that consist of two parts: a parallel gripper and a finger with SCARA arm kinematics (Chap. 8 in [22]), that we call the SCARA finger.

\section{1) Parallel gripper}

- Holding an object strongly and robustly. Heavy objects can be held in place using a supporting table.

- Robustly performing grasping of middle-size objects.

- The parallel gripper could contain a joint-less finger if implemented as one fixed finger and a closing one. For the implemented prototype, we chose a symmetric moving parallel gripper, but in the 

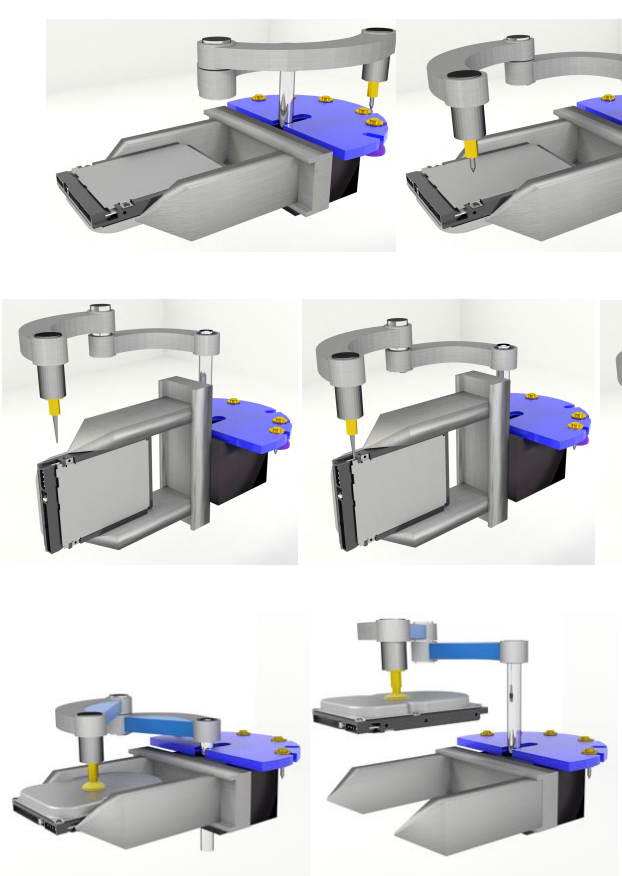

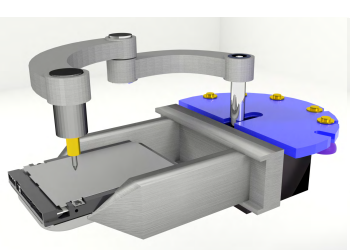

(a)
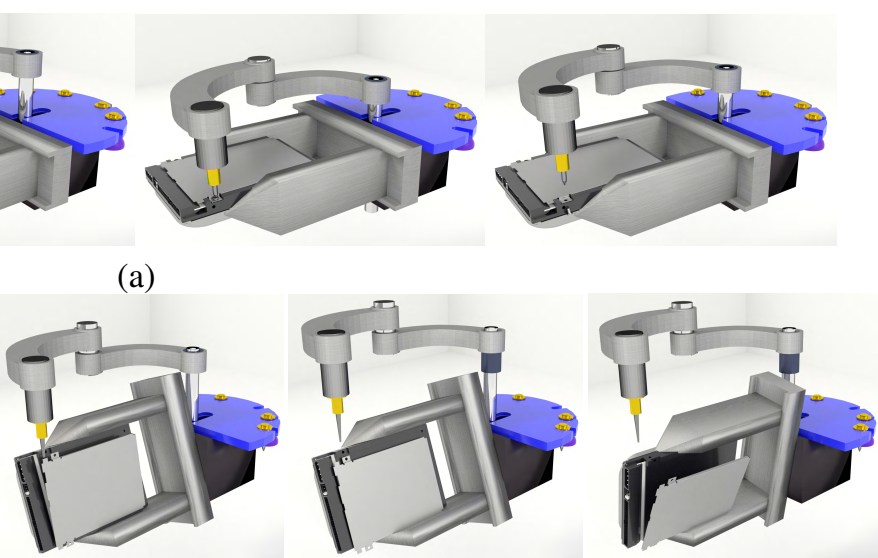

(b)
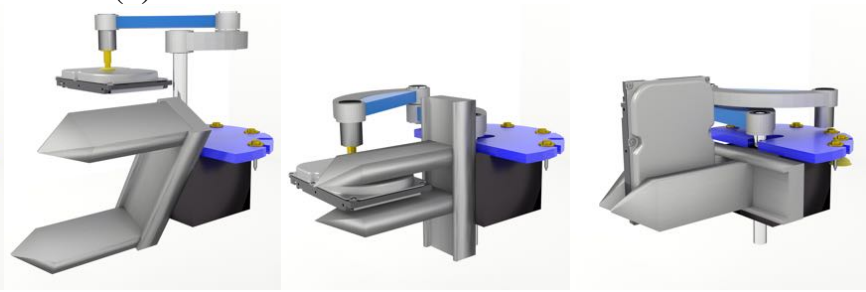

(c)

Fig. 3: Disassembly actions such as (a) unscrewing the screws of a HDD cover or (b) levering out an unscrewed HDD cover, where the rotational movement to lever is achieved by moving the grasped object with the parallel gripper instead of rotating the tool. (c) shows an object in hand reorientation.

future, we will consider other implementations if pushing can't be realized with the tip of the closed parallel gripper.

\section{2) SCARA finger}

- Implementing the architecture of an industrial SCARA arm, consisting of a Prismatic-RevoluteRevolute kinematic chain to serve different functionalities

- Act as a third finger for certain power grasps and as extra support for cylindrical grasps

- Pick up tools stored in the gripper base using an ad-hoc smart design that utilizes a suction actuation and the profile geometries of the tools to fit in the tool holder of the SCARA finger.

- Work on the object held by the parallel gripper using the available tools.

- The tool holder of the SCARA finger has the ability to rotate continuously around the tool axis, like electrical screwdrivers do.

- The two last links of the SCARA finger are covered with soft material for additional grasping functionality.

The multi-functionality of our gripper results from the multitude of available tools it can operate with. For the initial prototype, we will focus on a screwdriver tool, a levering tool and a suction gripper tool. For instance, we are considering the addition of a gripper tool similar to the gripper proposed in [23] utilizing the suction actuation already implemented in the SCARA finger. This would allow to grasp small odd-shaped objects such as loose screws. In future developments, many other tools can be added, allowing multiple possibilities to manipulate objects and operate on them for different purposes.

The kinematic structure has a total of 6 degrees of freedom, two for the parallel gripper, 3 for the SCARA finger and one extra rotation for the tool holder. The structure is shown in Fig. 2, where:

- Rotational joint $\gamma_{1}$ : Controls the rotation of the parallel gripper around the $y$-axis.

- Prismatic joint $\gamma_{2}$ : Controls the opening of the parallel gripper that moves symmetrically.

- Prismatic joint $\lambda_{1}$ : It is the first joint of the SCARA finger.

- Rotational joints $\lambda_{2}, \lambda_{3}$ : Correspond to the second and third joints of the SCARA finger, both are rotational joints acting on the same plane.

- Rotational joint $\lambda_{4}$ : Tool holder that can continuously rotate around the tool axis.

The concept was initially created using a 3D graphics software to show the design principles and the manipulation actions that can be performed (Section IV-A). Later, the kinematic and physics models was imported into Simox [24] to analyze general purpose grasping in quasi-static simulation Section IV-B and finally, an implementation proposal is shown in Section V. 


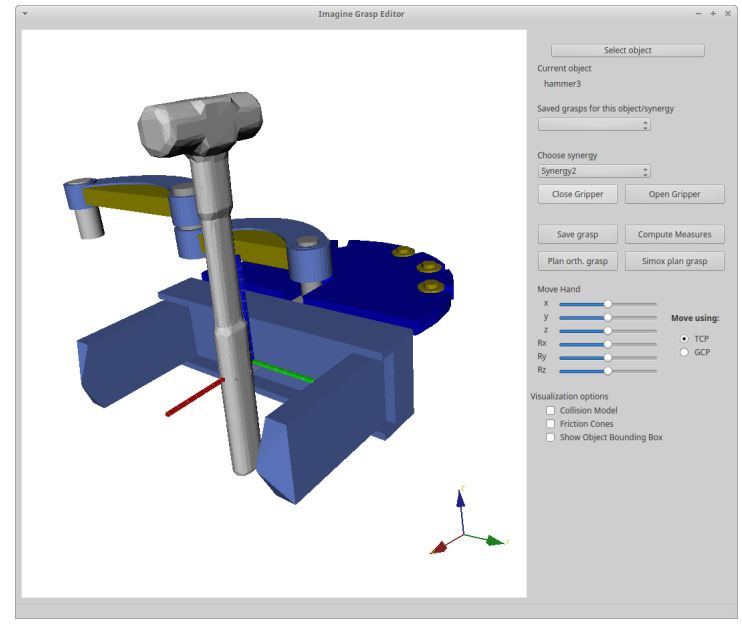

Fig. 4: Simox software showing the grasping computation tool. It allows to choose between different objects, synergies to open and close around the objects and computes different quality metrics.

\section{CAPAbILities OF THE KIT SWISS-KNIFE GRIPPER}

\section{A. Dexterous bimanual manipulations with the gripper}

The main design objective of the proposed gripper is to be able to perform bimanual manipulation actions for disassembly tasks. We particularly focused on disassembly of hard drives which includes complex tasks like unscrewing and levering to remove a cover. In Fig. 3 we show how we have planned the actions of unscrewing and levering, which are also visualized in the attached video. Unscrewing, shown in Fig. 3-(a), is realized by picking up the screwdriver tool, fitting the tool on the screw and rotating the tool holder around the tool axis of the tool. Please, note that the perception process to localize the screw is considered in the project but is out of the scope of the paper. The action of levering, shown in Fig. 3-(b), has to include a rotational movement that can not be realized with the proposed kinematics of the SCARA finger. As a solution, we propose to use the levering tool, insert it into the gap where we want to apply the levering action using the SCARA finger, and then use the rotational degree of freedom of the parallel gripper to execute the rotational movement of the levering action. Fig. 3-(c) shows the process of in-hand reorientation of a grasped object using the suction gripper tool. All the actions are shown in the attached video.

\section{B. Grasping with the KIT Swiss-Knife gripper}

The mesh model shown in Fig. 1 was exported and defined as a Simox model for the study of quasi static force closure grasps [24]. Simox is a grasp simulator similar to GraspIt [25] that detects collisions with objects during hand closure. The setup framework is shown in Fig. 4 For each given object, a grasp is defined as the location of the hand with respect to the object and the hand closure strategy (synergy).

We have defined three different strategies to open and close the hand, that we call synergies 1,2 and 3:

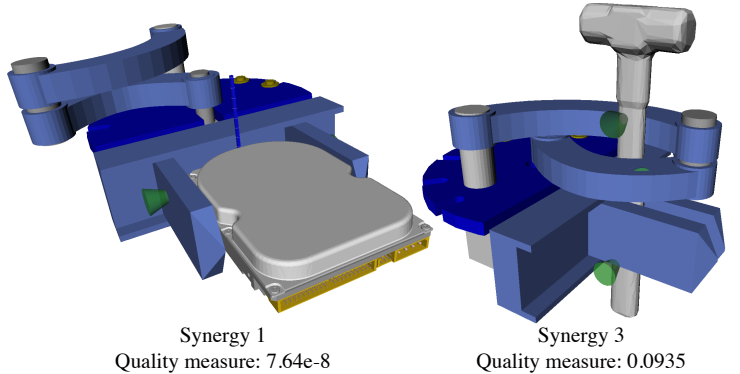

(a)

(b)

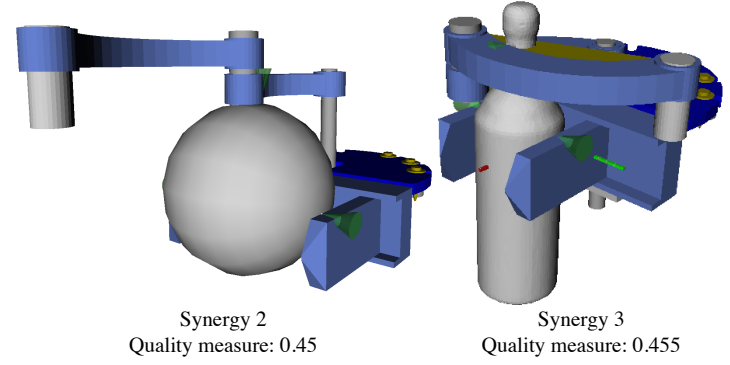

(c)

(d)

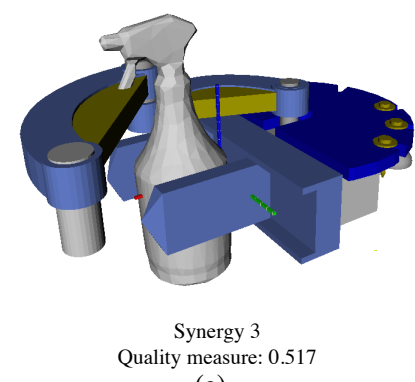

(e)

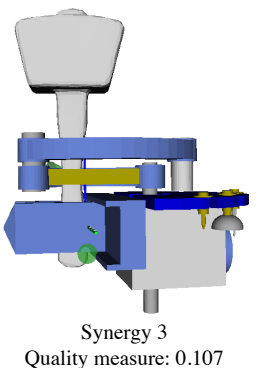

(f)
Fig. 5: Resulting force closure grasps using different synergies, together with the wrench space quality measure computed by Simox. Contact points are shown with their friction cones in green.

1) Parallel gripper (ignoring the SCARA finger)

- Parallel gripper closes: $\gamma_{1}$ fixed, $\gamma_{2}$ from max to 0

- SCARA finger: Three joints static. $\lambda_{1}=\lambda_{2}=$ $\lambda_{3}=0$ in closed position as shown in Fig. 5. (a).

2) Parallel 3 finger griper (SCARA finger as a third finger)

- Parallel gripper closes: $\gamma_{2}$ from max to 0 .

- Prismatic joint $\lambda_{1}$ goes from max to 0 .

- Fixed joints $\lambda_{2}=\lambda_{3}=\frac{\pi}{2}$ corresponding to fully extended arm. Other arm configurations could be explored for better grasp support.

3) Parallel gripper using SCARA finger for lateral grips

- Parallel gripper closes: $\gamma_{2}$ from max to 0 .

- Prismatic joint static at a configurable hight $\lambda_{1}=$ $h_{0}$.

- Joints $\lambda_{2}$ moves from 0 to $\frac{\pi}{2}$ and $\lambda_{3}$ from 0 to $\pi$. Different ratio of speeds between $\lambda_{2}$ and $\lambda_{3}$ should be tested.

The implementation of these 3 synergies in Simox allowed us to test the gripper capabilities with object shapes and different grasping types. Fig. 5 shows force closure grasps 


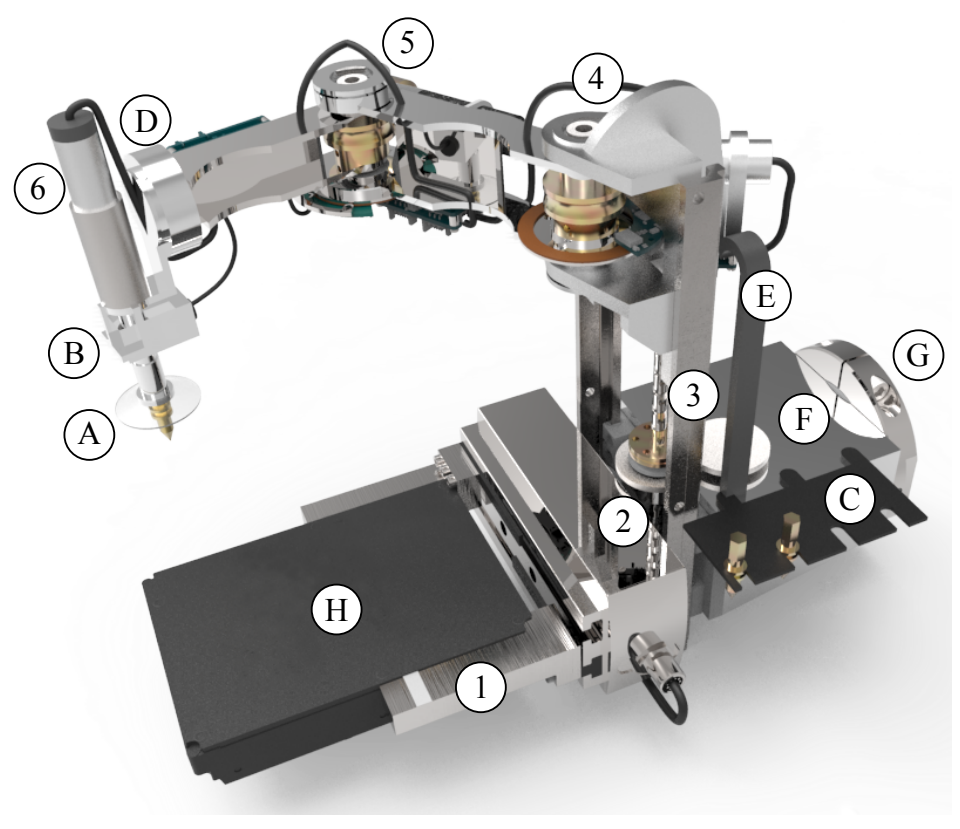

\begin{tabular}{cl}
\hline Comp. & Description \\
\hline 1 & $\begin{array}{l}\text { Motor gripper jaws } \\
\text { Motor gripper rotation } \\
\text { Motor prism. joint } \\
\text { (SCARA finger) }\end{array}$ \\
& $\begin{array}{l}\text { Motor 1st rot. joint } \\
\text { (SCARA finger) }\end{array}$ \\
& Motor 2nd rot. joint \\
5 & (SCARA finger) \\
6 & Motor tool rotation \\
\hline A & Pneumatic suction tool \\
& with bit \\
B & $\begin{array}{l}\text { Pneumatic feed-through } \\
\text { (continuous rotation) }\end{array}$ \\
C & Tool storage \\
D & Force-torque-sensor \\
E & Power chain (for cabling) \\
F & Base \\
G & (with hidden electronics) \\
H & Arm adapter \\
\hline
\end{tabular}

Fig. 6: Rendering of the CAD design for the first prototype of the KIT Swiss-Knife gripper with description of the individual components.

computed with Simox using the different synergies to close the hand, indicating also the grasp quality measure. Simox uses the normalized grasp wrench space volume as a quality measure, which gives an idea of how big is the wrench space generated through the contact forces (valued from 0 to 1 , being 1 the optimum but rarely realized). Note that the foam in the SCARA finger links is ignored for Simox collision checking, because Simox does not handle soft materials.

Fig. 5 shows the potential versatility of the SCARA finger for achieving different grasps. Synergy 2 enables the gripper for power grasping while synergy 3 can achieve more stable cylindrical grasps (as compared to only using the parallel gripper), but also operate articulated objects like shown in Fig. 5-(e).

\section{CAD DESIGN}

The CAD implementation of a first prototype using the presented concept has been developed with respect to all functional and operational requirements and it is shown in Fig. 6. It is designed to grasp and manipulate hard disk drives (HDDs) in different sizes $\left(2.5^{\prime \prime}\right.$ and $\left.3.5^{\prime \prime}\right)$ as well as all electronic devices which are smaller in size. The range of the joints is limited, without affecting the functionality of the gripper. The suction tool used to lift and re-orientate the grasped object and the tool changing mechanism are both integrated in one component, using the same pneumatic pipe (Fig. 7). To change the tools, the tool holder moves over a tool in the tool storage and then applies a vacuum to hold it. The tools themselves have a hexagonal profile as an adapter for the tool holder, so torque can easily be transferred. Their geometry is therefore similar to bits for common screwdrivers. Tools with diverse tips and utilities, e. g. cross recessed screwdrivers, slotted screwdrivers, levering tools, hooks or spikes can be mounted. A pneumatic feed-through between the motor and the tool changer allows continuous rotation which is needed for unscrewing. The suction cup for the suction gripper is mounted at the end of the tool holder. When no tool is plugged into the tool holder, the suction cup can be placed on a plane surface and vacuum can be applied. This allows the suction gripper to be used without having to pick up a separate tool.

The required forces $F$ and torques $T$ on the structure and joints (Table II) are derived from the functional and operational requirements (Table I):

- Grasping actions as pick up, grasp, pick to uncover/remove, rattle, shake, bang, break off are performed by the robotic arm and gripper rotation. Most of the required forces $F$ and torques $T$ were determined in experiments in which a human performed these tasks. As measuring instruments, we mainly used dynamometers. Considering the necessary force for closing the gripper, a test rig for the gripper was built (Fig. 8): The HDD was clammed between two gripper jaws with a force of $20 \mathrm{~N}$, induced by a weight $(2 \mathrm{~kg})$, while it was manually disassembled by a human. Due to the similar load case, we assume the same requirement for the SCARA finger grip, which determines the minimum torque of the 2 nd arm joint $(1.3 \mathrm{~N} \mathrm{~m})$. 
TABLE I: Functional and operational requirements

\begin{tabular}{lll}
\hline Category & \multicolumn{1}{c}{$\begin{array}{c}\text { Action or } \\
\text { case of loading }\end{array}$} & $\begin{array}{l}\text { Force/ } \\
\text { Torque }\end{array}$ \\
\hline Grasping actions & $\begin{array}{l}\text { Closing the gripper } \\
\text { SCARA finger grip }\end{array}$ & $20 \mathrm{~N}$ \\
& $20 \mathrm{~N}$ \\
\hline Pushing actions & See comment below & \\
\hline Actions with tools & Pulling or pushing & $10 \mathrm{~N}$ \\
& Unscrewing & $1 \mathrm{~N} \mathrm{~m}$ \\
& Levering & $5 \mathrm{~N}$ \\
\hline Operational req. & Lifting SCARA finger & $10 \mathrm{~N}$ \\
& Lifting SCARA finger and HDD & $16 \mathrm{~N}$ \\
& Gravity (orientation dependent) & \\
& Moments of inertia & \\
\hline
\end{tabular}

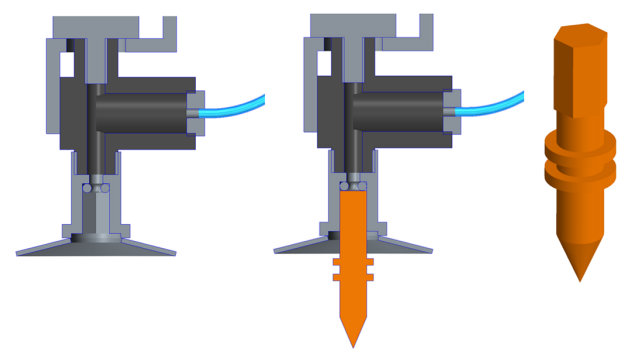

Fig. 7: The suction tool without (left) and with (middle) plugged in tool as tool holder, tool geometry (right)

- Pushing actions only have a very low impact on the forces and torques occurring in the joints and structure of the gripper. To support the forces effected by pushing actions, we use a rigid structure. The robotic arm, on which the gripper is mounted, performs most of the actions needed. The gripper jaws can close completely to provide a centered pushing tool.

- Actions with tools describe torques and forces, that only occur when using certain tools. Similar to the grasping actions, these values were determined in tests, in which a human disassembled the HDD. Again, we mainly used dynamometers to determine the force. However, in the case of the necessary torque for unscrewing $(1 \mathrm{Nm})$, we used a torque wrench.

- Operational requirements are torques, which counteract moments of inertia as well as gravity-induced torques and forces. General requirements in this case are gravity forces induced by the weight of the SCARA finger $(10 \mathrm{~N})$ and the maximum weight of the HDD $(6 \mathrm{~N})$. To enable working in different orientations, we also considered the gravity-induced torques in a $90^{\circ}$ rotation of the entire system. This case represents the required torque for the $1 \mathrm{st}$ arm $(1.5 \mathrm{Nm})$, which in standard orientations only has to overcome moments of inertia and friction.

Table II lists all joint specifications for the chosen motorgearbox combinations. Apart from the combined suction gripper and tool holder, all joints are electrically actuated. For all rotary joints, we use Maxon DCX 22S motors and planetary gears (ratio $i=231$ ). The prismatic joint is realized by a Maxon DCX $16 \mathrm{~S}$ motor in combination with a planetary gear, a belt drive, a spindle (lead $2.54 \mathrm{~mm}$ ) and guiding rails that absorb the bending forces. For the parallel gripper we foresee the electrically actuated FESTO HGPE 14-60. To ensure transferability to the gripper and different objects, we use safety factors $S \geq 3$ based on the maximum forces $F_{\max }$ or torques $T_{\max }$. For controlling the gripper's movements, relative and absolute position encoders (Renishaw AksIM MBA 7) are integrated in the construction as well as a 6d-force-torque-sensor (ATI Mini40). The KIT Swiss Knife Gripper is supposed to be attached to a robot arm as endeffector. This is why the prototype foresees an standard arm adapter (Schunk FWA 115), which connects the gripper with the robot arm mechanically as well as electronically. Since not only the gripper but also the objects in the gripper have to be lifted up by the robot arm, a rigid lightweight construction made of high-strength aluminum alloy is implemented. The final weight will be approximately $2 \mathrm{~kg}$.

\section{DISCUSSION}

The present work serves as preliminary work towards the implementation of a fully functional prototype. Therefore, there are some design choises that will have to be tested and compared in more detail

For instance, the parallel gripper could be substituted by any other planar gripper acting on the same plane. If instead of a parallel gripper we implement a couple of underactuated fingers, we could increase the flexibility and the ability to grasp many different objects shapes. However, an underactuated gripper may not be able to hold the object strongly enough to perform accurate actions on it with the SCARA finger. An industrial two finger gripper with two revolute joints could achieve the desired stability and allow to open the gripper completely in a way that the SCARA finger could also be used alone to operate on a bigger object.

Similarly, the SCARA finger could be implemented with different kinematic structure, like a 3R chain or a RPP chain, still following the same idea of bimanual task gripper. During the development of different prototypes, all these options will be explored.

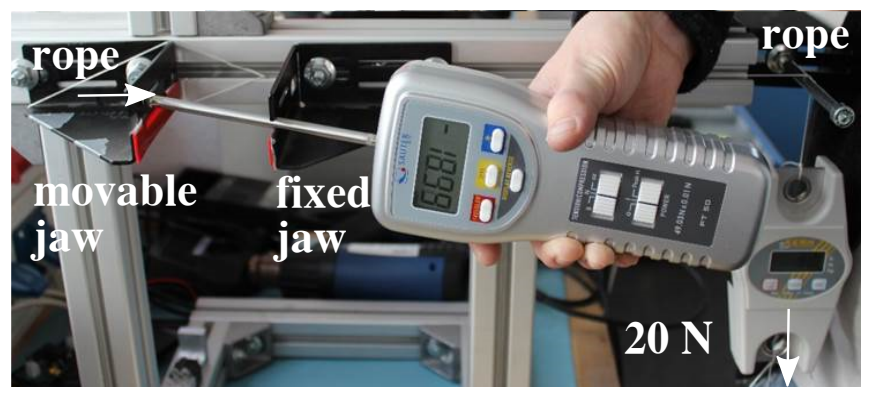

Fig. 8: Test rig to determine the required force for closing the gripper: A weight of $2 \mathrm{~kg}$ is converted into a force of $20 \mathrm{~N}$ that actuates the left gripping jaw via a rope mechanism 
TABLE II: Requirements of the joints and specification of the first CAD prototype

\begin{tabular}{|c|c|c|c|c|c|c|c|}
\hline Joint & $\begin{array}{l}\text { Required } \\
F \text { or } T\end{array}$ & $\begin{array}{l}\text { Realized } \\
F_{n} \text { or } T_{n}\end{array}$ & $\begin{array}{l}\text { Realized } \\
F_{\max } \text { or } T_{\max }\end{array}$ & $\begin{array}{l}\text { Speed } \\
v \text { or } n\end{array}$ & $\begin{array}{l}\text { Range of } \\
\text { motion }\end{array}$ & $\begin{array}{l}\text { Arm } \\
\text { length }\end{array}$ & $\begin{array}{l}\text { Absolute } \\
\text { accuracy }\end{array}$ \\
\hline $\begin{array}{l}\text { Parallel } \\
\text { gripper }\end{array}$ & $20 \mathrm{~N}$ & $60 \mathrm{~N}$ & $120 \mathrm{~N}$ & $30 \mathrm{~mm} / \mathrm{s}$ & $0 \mathrm{~mm}$ to $120 \mathrm{~mm}$ & & None \\
\hline $\begin{array}{l}\text { Gripper } \\
\text { rotation }\end{array}$ & $0.5 \mathrm{~N} \mathrm{~m}$ & $3.0 \mathrm{~N} \mathrm{~m}$ & $5.3 \mathrm{~N} \mathrm{~m}$ & $104^{\circ} / \mathrm{s}$ & $-90^{\circ}$ to $+90^{\circ}$ & & $<0.1^{\circ}$ \\
\hline $\begin{array}{l}\text { Prismatic } \\
\text { joint }\end{array}$ & $16 \mathrm{~N}$ & $40 \mathrm{~N}$ & $160 \mathrm{~N}$ & $15.9 \mathrm{~mm} / \mathrm{s}$ & $0 \mathrm{~mm}$ to $75 \mathrm{~mm}$ & & $0.1 \mathrm{~mm}$ \\
\hline 1st arm & $1.5 \mathrm{~N} \mathrm{~m}$ & $2.5 \mathrm{~N} \mathrm{~m}$ & $4.5 \mathrm{~N} \mathrm{~m}$ & $278^{\circ} / \mathrm{s}$ & $-120^{\circ}$ to $100^{\circ}$ & $110 \mathrm{~mm}$ & $<0.1^{\circ}$ \\
\hline 2nd arm & $1.3 \mathrm{Nm}$ & $2.5 \mathrm{~N} \mathrm{~m}$ & $4.5 \mathrm{~N} \mathrm{~m}$ & $278^{\circ} / \mathrm{s}$ & $0^{\circ}$ to $155^{\circ}$ & $110 \mathrm{~mm}$ & $<0.1^{\circ}$ \\
\hline Tool & $1.0 \mathrm{~N} \mathrm{~m}$ & $2.2 \mathrm{~N} \mathrm{~m}$ & $3.3 \mathrm{Nm}$ & $278^{\circ} / \mathrm{s}$ & $\pm \infty$ & & None \\
\hline
\end{tabular}

\section{CONCLUSION AND Future Work}

We presented the concept of a gripper for disassembly tasks that has the potential to provide solutions for several gaps in the literature of robotic grippers: it solves the problem of grabbing tools in a smart way providing an autonomous tool change system and it implements the idea of devoting some fingers at grasping and some fingers at performing manipulations on the grasped object, being de facto a bimanual system embedded in a single gripper that only requires a single robotic arm.

The concept has been presented and tested in static simulation. We tested the force requirements for the tasks, and presented a CAD design that serves as a possible implementation of a functional prototype. In the near future work, we will build a prototype and test the gripper abilities in the context of disassembly tasks.

\section{REFERENCES}

[1] J. R. Duflou, G. Seliger, S. Kara, Y. Umeda, A. Ometto, and B. Willems, "Efficiency and feasibility of product disassembly: A casebased study," CIRP Annals, vol. 57, no. 2, pp. 583-600, 2008.

[2] C. Rujanavech, J. Lessard, S. Chandler, S. Shannon, J. Dahmus, and R. Guzzo, "Liam - an innovation story." https://www.apple.com/environment/pdf/Liam_white_paper_Sept2016.pdf 2016.

[3] J. Cui and E. Forssberg, "Mechanical recycling of waste electric and electronic equipment: a review," Journal of hazardous materials, vol. 99, no. 3, pp. 243-263, 2003.

[4] R. R. Ma and A. M. Dollar, "On dexterity and dexterous manipulation," in 15th International Conference on Advanced Robotics (ICAR), pp. 1-7, 2011.

[5] M. Mason, S. Srinivasa, and A. Vazquez, "Generality and simple hands," Robotics Research, pp. 345-361, 2011.

[6] K. Tai, A.-R. El-Sayed, M. Shahriari, M. Biglarbegian, and S. Mahmud, "State of the art robotic grippers and applications," Robotics, vol. 5, no. 2, p. 11, 2016

[7] J. Borràs and A. M. Dollar, "Dimensional synthesis of three-fingered robot hands for maximal precision manipulation workspace," The International Journal of Robotics Research, vol. 34, no. 14, pp. 1731$1746,2015$.

[8] C. Smith, Y. Karayiannidis, L. Nalpantidis, X. Gratal, P. Qi, D. V. Dimarogonas, and D. Kragic, "Dual arm manipulation-a survey," Robotics and Autonomous systems, vol. 60, no. 10, pp. 1340-1353, 2012.

[9] K. Nagata, "Manipulation by a parallel-jaw gripper having a turntable at each fingertip," in IEEE International Conference on Robotics and Automation, pp. 1663-1670, 1994.
[10] G. Grunwald, C. Borst, J. Zöllner, et al., "Benchmarking dexterous dual-arm/hand robotic manipulation," in IEEE/RSJ International Conference on Intelligent Robots and Systems, Workshop on Performance Evaluation and Benchmarking, 2008.

[11] N. Rahman, L. Carbonari, M. D'Imperio, C. Canali, D. G. Caldwell, and F. Cannella, "A dexterous gripper for in-hand manipulation," in IEEE International Conference on Advanced Intelligent Mechatronics (AIM), pp. 377-382, 2016.

[12] A. Bicchi and A. Marigo, "Dexterous grippers: Putting nonholonomy to work for fine manipulation," The International Journal of Robotics Research, vol. 21, no. 5-6, pp. 427-442, 2002.

[13] R. R. Ma and A. M. Dollar, "An underactuated hand for efficient finger-gaiting-based dexterous manipulation," in IEEE International Conference on Robotics and Biomimetics (ROBIO), pp. 2214-2219, 2014

[14] J. Schmitt, H. Haupt, M. Kurrat, and A. Raatz, "Disassembly automation for lithium-ion battery systems using a flexible gripper," in 15th International Conference on Advanced Robotics (ICAR), pp. 291-297, IEEE, 2011.

[15] C. Canali, F. Cannella, F. Chen, T. Hauptman, G. Sofia, D. Caldwell, and A. Eytan, "High reconfigurable robotic gripper for flexible assembly," in ASME International Design Engineering Technical Conferences and Computers and Information in Engineering Conference.

[16] A. Weigl-Seitz, K. Hohm, M. Seitz, and H. Tolle, "On strategies and solutions for automated disassembly of electronic devices," The International Journal of Advanced Manufacturing Technology, vol. 30, no. 5-6, pp. 561-573, 2006.

[17] R. Molfino, R. P. Razzoli, and M. Zoppi, "A low-cost reconfigurable gripper for assembly and disassembly tasks in white industry," IFAC Proceedings Volumes, vol. 39, no. 15, pp. 498-505, 2006.

[18] G. Seliger, T. Keil, U. Rebafka, and A. Stenzel, "Flexible disassembly tools," in IEEE International Symposium on Electronics and the Environment, pp. 30-35, 2001.

[19] A. ElSayed, E. Kongar, S. M. Gupta, and T. Sobh, "A robotic-driven disassembly sequence generator for end-of-life electronic products," Journal of Intelligent \& Robotic Systems, vol. 68, no. 1, pp. 43-52, 2012.

[20] K. Wegener, W. H. Chen, F. Dietrich, K. Dröder, and S. Kara, "Robot assisted disassembly for the recycling of electric vehicle batteries,' Procedia CIRP, vol. 29, pp. 716-721, 2015.

[21] W. H. Chen, K. Wegener, and F. Dietrich, "A robot assistant for unscrewing in hybrid human-robot disassembly," in IEEE International Conference On Robotics and Biomimetics, pp. 536-541, 2014.

[22] J. J. Craig, Introduction to robotics: mechanics and control, vol. 3. Pearson/Prentice Hall Upper Saddle River, NJ, USA:, 2005.

[23] J. R. Amend, E. Brown, N. Rodenberg, H. M. Jaeger, and H. Lipson, "A positive pressure universal gripper based on the jamming of granular material," IEEE Transactions on Robotics, vol. 28, no. 2, pp. 341-350, 2012.

[24] N. Vahrenkamp, M. Kröhnert, S. Ulbrich, T. Asfour, G. Metta, R. Dillmann, and G. Sandini, "Simox: A robotics toolbox for simulation, motion and grasp planning," in International Conference on Intelligent Autonomous Systems (IAS), 2012.

[25] A. T. Miller and P. K. Allen, "Graspit! a versatile simulator for robotic grasping," IEEE Robotics \& Automation Magazine, vol. 11, no. 4, pp. 110-122, 2004 